\title{
Spatial Sampling to Detect an Invasive Pathogen Outside of an Eradication Zone
}

\author{
I. Demon, N. J. Cunniffe, B. P. Marchant, C. A. Gilligan, and F. van den Bosch
}

First, third, and fifth authors: Department of Biomathematics and Bioinformatics, Rothamsted Research, West Common, Harpenden, Hertfordshire, AL5 2JQ, United Kingdom; and second and fourth authors: University of Cambridge, Department of Plant Sciences, Downing Site, Cambridge, CB2 3EA, United Kingdom.

Accepted for publication 13 January 2011.

\begin{abstract}
Demon, I., Cunniffe, N. J., Marchant, B. P., Gilligan, C. A., and van den Bosch, F. 2011. Spatial sampling to detect an invasive pathogen outside of an eradication zone. Phytopathology 101:725-731.

Invasive pathogens are known to cause major damage to the environments they invade. Effective control of such invasive pathogens depends on early detection. In this paper we focus on sampling with the aim of detecting an invasive pathogen. To that end, we introduce the concept of optimized spatial sampling, using spatial simulated annealing, to plant pathology. It has been mathematically proven (15) that this optimization

easily be applied for disease detection, and then we show that (ii) combining it with a spatially explicit epidemic model, we can develop optimum sample schemes, i.e., optimum locations to sample that maximize the probability of detecting an invasive pathogen. This method is then used as baseline against which other sampling methods can be tested for their accuracy. For the specific example case of this paper, we test (i) random sampling, (ii) stratified sampling as well as (iii) sampling based on the output of the simulation model (using the most frequently infected hosts as sample points), and (iv) sampling the hosts closest to the outbreak point.
\end{abstract} method converges to the optimum allocation of sampling points that give the largest detection probability. We show the benefits of the method to plant pathology by (i) first illustrating that optimized spatial sampling can
Additional keywords: dispersal kernel, objective function, SEIR model.
As a result of international free trade, there is an increased risk of introduction of invasive and nonindigenous pathogens (13). There have been numerous invasions that caused major ecological and economic damage. Pimentel et al. (25) estimate that approximately $\$ 21$ billion in crop losses per year are attributable to nonindigenous plant pathogens in the United States, in addition to control costs of approximately $\$ 500$ billion per year. In the UK, Jones and Baker (14) showed that more than 234 pathogens were introduced between 1970 and 2004. Important recent examples include Dutch elm disease caused by the fungal pathogen Ophiostoma novo ulmi, rhizomania of sugar beet caused by Beet necrotic yellow vein virus (BNYVV), and sudden oak death caused by Phytophthora ramorum $(2,4,5,9)$. The nursery trade is one of the possible routes of entry for invasive plant pathogens via the inadvertent import of infected plant stock $(4,14)$. This paper deals with a pathogen that has entered through this route. The pathogen can subsequently disperse into the surrounding natural environment, where there is potential for significant ecological damage. Consequently, a major challenge for regulatory agencies is the early detection of the pathogen to prevent its spread into the surrounding area.

Quarantine measures as well as eradication or containment plans are applied or are being developed for potential invader pathogens. It is well known that eradication or containment can only be successful when the invader is detected at an early stage of the epidemic it causes $(11,23,24,29)$. Therefore it is of key

Corresponding author: F. van den Bosch

E-mail address: frank.vandenbosch@bbsrc.ac.uk

* The $\boldsymbol{e}$-Xtra logo stands for "electronic extra" and indicates that Figures 1 and 3 appear in color online.

doi:10.1094/PHYTO-05-09-0120

(C) 2011 The American Phytopathological Society importance for those pathogens entering through the trade network that a plan is in place to sample the area around a nursery or retail park when the pathogen is detected there. Although sampling with the aim of detection has been a key focus in studies of rare animal and plant species (7), it has received little attention in the field of plant disease epidemiology, where interest has been directed towards estimating disease incidence of pathogens that are known to be present $(12,17-20,21)$.

In this paper, we focus on sampling to detect a pathogen that may have escaped from a zone of eradication. We consider the scenario where a pathogen has been locally eradicated from a known site of introduction (e.g., within a nursery or garden centre) and we want to determine whether it has escaped to the surrounding environment. We focus specifically on the invasive pathogen $P$. ramorum in the UK to motivate the problem. $P$. ramorum is currently of statutory concern (through EU regulations on its spread in the EU) in the UK, as it poses a threat to woodlands $(1,10)$. Arguably, most outbreaks in the UK started within nurseries and garden centers, where host species of $P$. ramorum, including Rhododendron ponticum and Viburnum spp., are cultivated $(1,27)$. It has been detected in some woodlands, where Britain's two native (susceptible) oak tree species, Quercus robur and $Q$. petraea occur, are interspersed with $R$. ponticum in some locations $(1,6)$. In an effort to minimize the ecological damage caused by $P$. ramorum, the woodlands are continuously surveyed and rhododendron is removed from woodlands on detection (8). We introduce spatial optimization using simulated annealing techniques to plant pathology and use it to generate sampling plans that maximize the probability to detect the pathogen (15). It is a technique widely used in geostatistics and has been mathematically proven to yield the sampling plan that maximizes the probability to detect (15). We will describe the logic behind the method in the section entitled optimized spatial sampling using simulated annealing. 
The use of the optimization method can only be shown by way of an example. Therefore, we develop a spatially explicit SEIR stochastic simulation model and use it to generate multiple epidemic realizations. As discussed, the method guarantees the sampling scheme that maximizes the probability to detect the disease. It has the disadvantage, however, that it requires the development of an epidemic model, multiple simulation runs, and the application of the optimization technique for it to be applied on a case by case basis. What we will do as a next step is to use the optimum sampling plan to test how accurate other, simpler to use, sampling designs are compared to the optimum solution. If a simple method to develop a sampling plan performs close to that of the optimum solution, it can be a useful candidate for application in routine field-based sampling plans. We will compare optimal sampling plans with methods such as (i) sampling schemes based directly on the probability of disease occurrence from simulated epidemics without formal optimization (hereafter referred to as probability map sampling), (ii) distance-based sampling, which entails sampling the $n$ hosts closest to the eradication zone, (iii) simple random sampling, and (iv) stratified random sampling.

\section{THEORY AND APPROACHES}

In the following sections we describe (i) the epidemic model, (ii) the optimized spatial sampling method, (iii) and the other sampling methods of which we assess the effectiveness, and (iv) we consider the effect of the epidemiological parameters and time elapsed since initial infection on the detection probability.

The epidemic model. We consider a spatially explicit stochastic model with hosts that are classified according to their disease status as susceptible ( $\mathrm{S}$ ), exposed but not yet infectious (E), infectious (I), and no longer infectious (removed) (R). The epidemic process depends on the epidemiological parameters and is defined by the transition probabilities between the disease classes (Table 1).

The epidemic model uses a kernel function, $K$, that describes the dispersal of inoculum about a source in two dimensions. This function is a probability distribution function, which has a dimension of inverse area. To calculate the probability of infection on a target plant this function is integrated over the area of the target host plant, $A_{i}$. It is a reasonable approximation, in most cases, to assume $K$ to be constant over the small area of a host plant. The transition probability, per time unit, for a healthy host to become infected, $\beta \Lambda_{i}$, or host $i$ to become infected thus is given by

$$
\beta \Lambda_{i}=\widetilde{\beta} A_{i} \sum_{j=1}^{N_{j}} K\left(d_{i j}\right)
$$

where $\beta=\widetilde{\beta} A_{i}$ is interpreted as the transmission rate parameter.

$$
\Lambda_{i}=\sum_{j=1}^{N_{j}} K\left(d_{i j}\right)
$$

TABLE 1. Summary of epidemiological parameters, variables, transition events, and the corresponding transition rates for the SEIR epidemic model

\begin{tabular}{ll}
\hline Variables & \multicolumn{1}{c}{ Description } \\
\hline $\mathrm{S}$ & Susceptible hosts \\
$\mathrm{E}$ & Exposed, latently infected hosts \\
$\mathrm{I}$ & Infectious hosts \\
$\mathrm{R}$ & Removed, noninfectious hosts \\
Parameters & The per capita transmission rate of disease \\
$\beta$ & The infection pressure for susceptible host $i$ \\
$\Lambda_{i}$ & The mean latent period \\
$1 / \theta$ & The mean infectious period \\
$1 / \gamma$ & Rate \\
Transition event & $\beta \Lambda_{i}$ \\
$\mathrm{~S} \Rightarrow \mathrm{E}$ & $\theta E$ \\
$\mathrm{E} \Rightarrow \mathrm{I}$ & $\gamma I$ \\
$\mathrm{I} \Rightarrow \mathrm{R}$ &
\end{tabular}

is termed the infection pressure, where $j$ is the subset of infectious plants, $N_{i}$ is the total number of infectious hosts, and $d_{i j}$ is the distance between susceptible host $i$ and infectious host $j$. This distance is calculated from

$$
d_{i j}=\sqrt{\left(x_{i}-x_{j}\right)^{2}+\left(y_{i}-y_{j}\right)^{2}}
$$

where $\left(x_{i}, y_{i}\right)$ are the Cartesian coordinates of host $i$.

To explore the effect of the shape of the dispersal kernel, we use two contrasting examples namely the thin tailed Gaussian kernel,

$$
K(d)=\frac{1}{2 \pi \sigma^{2}} \exp \left(-\frac{d^{2}}{2 \sigma^{2}}\right)
$$

and the thick tailed square root kernel,

$$
K(d)=\frac{\psi^{4}}{24 \pi} \exp (-\psi \sqrt{d})
$$

(Fig. 1A). We characterize these two dimensional, one parameter distributions by the mean distance, $d_{\text {mean }}$, a spore is deposited from the source. This mean distance is $d_{\text {mean }}=\sigma \sqrt{\pi / 2}$ and $d_{\text {mean }}=$ $20 / \psi^{2}$, respectively.

The probability per time unit that an exposed host plant becomes infectious is given by $\theta$; therefore, the mean duration of the latent period equals $1 / \theta$. An infectious host recovers with probability per unit time $\gamma$, with mean duration of the infectious period $1 / \gamma$. Using these transition probabilities, $P_{\text {transition, we draw }}$ for each host the time until this host would transit to the next stage, $T_{\text {transit }},(26)$ from

$$
T_{\text {transit }}=-\frac{1}{P_{\text {transition }}} \cdot \ln (\text { Rand })
$$

where Rand is a uniformly distributed random number between 0 and 1 . The host plant with the smallest $T_{\text {transit }}$ is selected and the corresponding transition event is performed.

Using the model, epidemics are simulated using biologically plausible values for the transmission rate, latent and infectious periods for the pathogen P. ramorum (A. R. Cook, C. A. Gilligan, R. K. Meentemeyer, R. C. Cobb, and D. M. Rizzo, personal communication). The parameter values used in each simulation are given in the figure subscripts. The dispersal parameters were arbitrarily selected to mimic dispersal in the range 0 to $10 \mathrm{~km}$ from the initial site of infestation. The host distribution considered here is a hypothetical distribution based on likely locations that reflect local topographical and soil conditions, for example clustering on edge of moors, in an area in the South West of the UK (Fig. 1B), where outbreaks have been reported $(4,6)$. We generate numerous runs of the epidemic model and for each run the epidemic is initiated in a cluster of 60 hosts mimicking a nursery/garden center (Fig. 1C). The sampling methods are applied to these realizations, which contain the status of each host within the host distribution at the end of the run time which is set at 100 days. Note that the location of the nursery or garden center is hypothetical and is for illustration only; it does not represent a real occurrence. Similarly, the distribution of susceptible hosts, while biologically plausible, is to illustrate the methods.

We assume that the sampling methods, described in the following sections, are implemented after detection and local eradication in the nursery/garden center. Therefore, only the host plants outside the eradication zone are sampled, for sample size $n=5,10,20$, and 40 . The number of simulated epidemic realizations for which at least one infected host plant is detected using the sampling schemes is divided by the total number of realizations evaluated to give the detection probability. This value is used as a comparative measure of the performance of the different sampling methods.

Optimized spatial sampling using simulated annealing. The sample of $n$ hosts that maximizes the detection probability of the 
pathogen can simply be found by calculating the detection probability obtained with all possible combinations of $n$ hosts and selecting the combination that maximizes the probability. However, this method is very time consuming due to the large number of possible combinations of $n$ host plants. We therefore use simulated annealing, an algorithm for global optimization problems originally developed in physics (15). The algorithm maximizes an
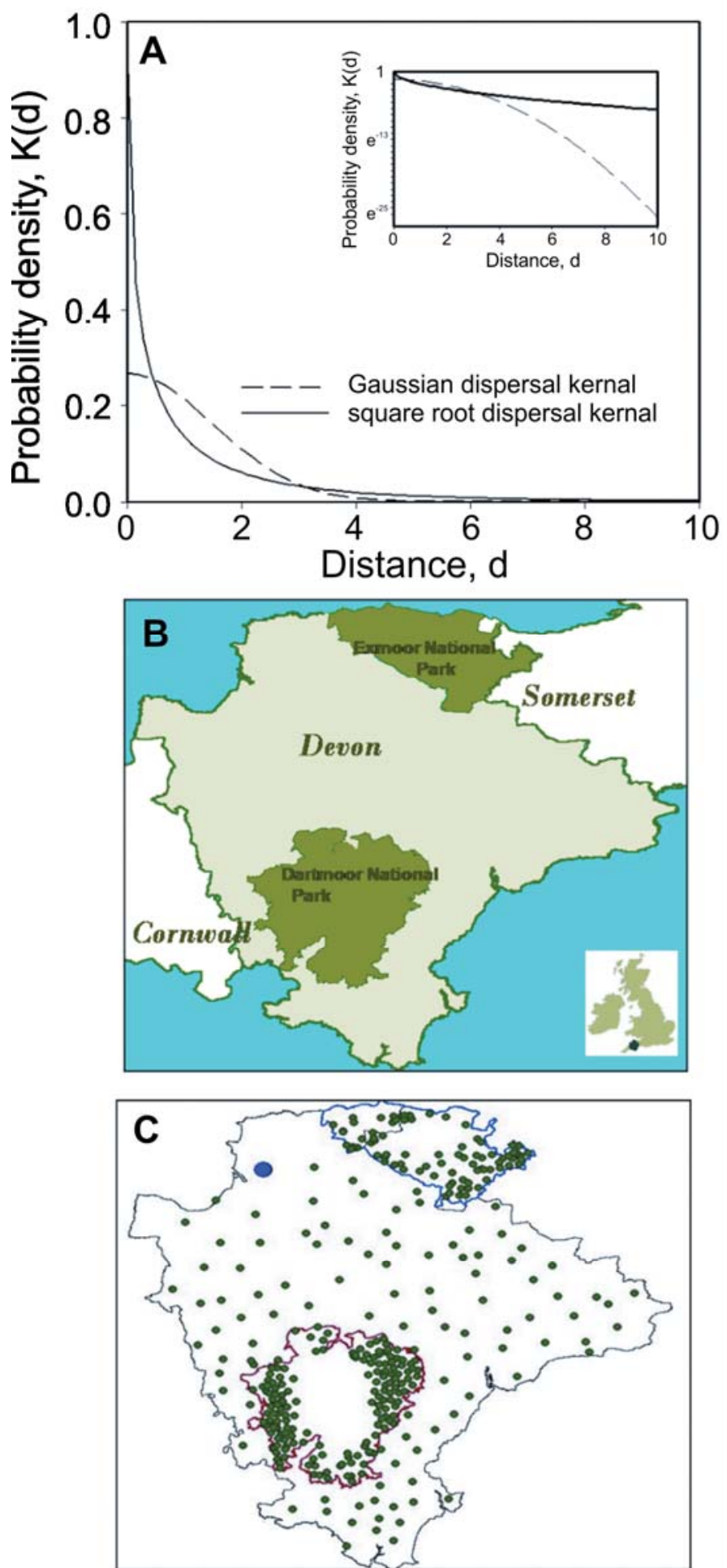

Fig. 1. A, The two pathogen dispersal kernels, K(d), used in the simulation model, with the graph inset showing a logarithmic transformation. B, Map of Devon, in the UK, on which the host distribution is based. C, Simulated host distribution. The small gray dots represent susceptible hosts and the large black dot is the hypothetical location of a nursery/garden center, the source of initial inoculum. The location of the nursery is randomly assigned and does not correspond with any known enterprise. objective function, $\Phi$, which in our case is the probability to detect an infected host.

The optimization method is an iterative process, proceeding in steps which will be denoted by the subscript $k$, that starts $(k=0)$ from a random sampling design by randomly selecting $n$ hosts from the host distribution. For this sample the probability to detect the host, $\Phi_{0}$, is calculated. Next $(k=1)$ we take one of the hosts in the sample and replace it with another host. The question then is whether this new set of $n$ hosts is a better sampling plan. To this end we calculate the probability to detect the epidemic for the new sample plan, $\Phi_{1}$. If $\Phi_{1}>\Phi_{0}$, then obviously the new sampling plan is better than the previous one and we retain this one. If $\Phi_{1} \leq \Phi_{0}$, the new sampling plan is not better than the old sampling plan. In this case the new sampling plan is still retained with probability $P$, where $P$ is given by

$$
P=\exp \left(\frac{\Phi_{k+1}-\Phi_{k}}{c_{k}}\right)
$$

This process is repeated. Every step where $\Phi_{k+1}>\Phi_{k}$, the new sampling plan is retained and every time $\Phi_{k+1} \leq \Phi_{k}$, the new sampling plan is retained with probability $P$ defined by Barr and Asher (2). It has been mathematically proven (15) that retaining inferior sampling plans with probability $P$ guaranties that the process converges to the global optimal sampling design (Fig. 2). The parameter $c_{k}$ decreases with $k$ as $c_{k+1}=\alpha \cdot c_{k}, \alpha \in[0,1](28)$. The parameter $c$ (called the cooling parameter) is crucial to the annealing process and its initial value is determined empirically through a series of simulations to ensure that the optimization process occurs gradually. The number of iterations required to reach the global maximum is determined empirically from several runs of the optimization process.

Here, the global maximum is the largest achievable probability for detection of disease across the epidemic realizations and the corresponding sample scheme is hereafter referred to as the optimum sample scheme. These optimum sample schemes are then applied to a new set of epidemic realizations (developed as described in the previous section) to test their efficiency in detecting the pathogen outside the eradication zone and in doing so we preclude any bias in the detection probability obtained with the optimum sampling schemes.

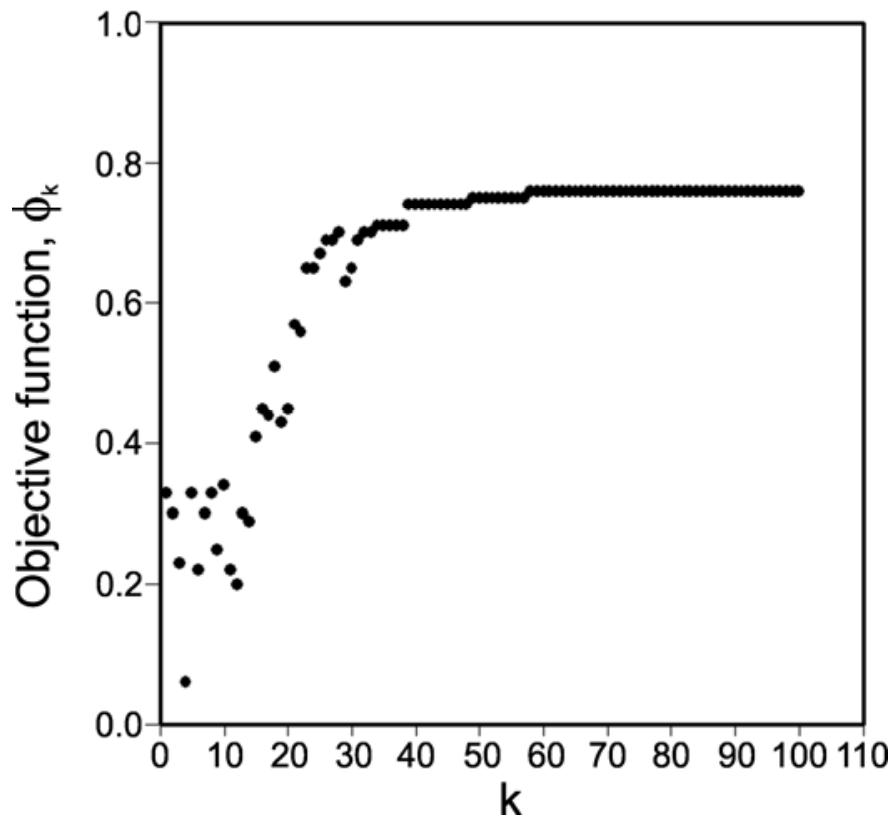

Fig. 2. The numerical value of the objective function, here the probability to detect the pathogen, $\Phi_{k}$, as a function of the number of iterations, $\mathrm{k}$ for a typical run of the process. 
Alternative sampling methods. We assess the performance of four other sampling methods. By doing so, we hope to find a sampling method that is easy to use in the field and at the same time approximates the detection probability of the optimum sampling scheme. We consider (i) probability map sampling based upon repeated simulations of the epidemic model, (ii) distancebased sampling, (iii) simple random sampling, and (iv) stratified random sampling. Note that the latter three sampling methods require only a map of susceptible hosts. They do not require an epidemic model from which to derive the sample scheme and can therefore be applied more readily.

The first sampling method involves sampling the $n$ hosts that have the largest probability of being infected across the entire set of simulated epidemics. This method is an obvious candidate to yield a sample scheme with a large detection probability. Distance-based sampling involves calculating the distance to the source of infection for all hosts outside the eradication zone and selecting the $n$ hosts closest to the source. Simple random sampling involves random selection of $n$ host plants to form a sampling scheme. For stratified sampling, the host distribution is divided into different strata according to increasing distance from
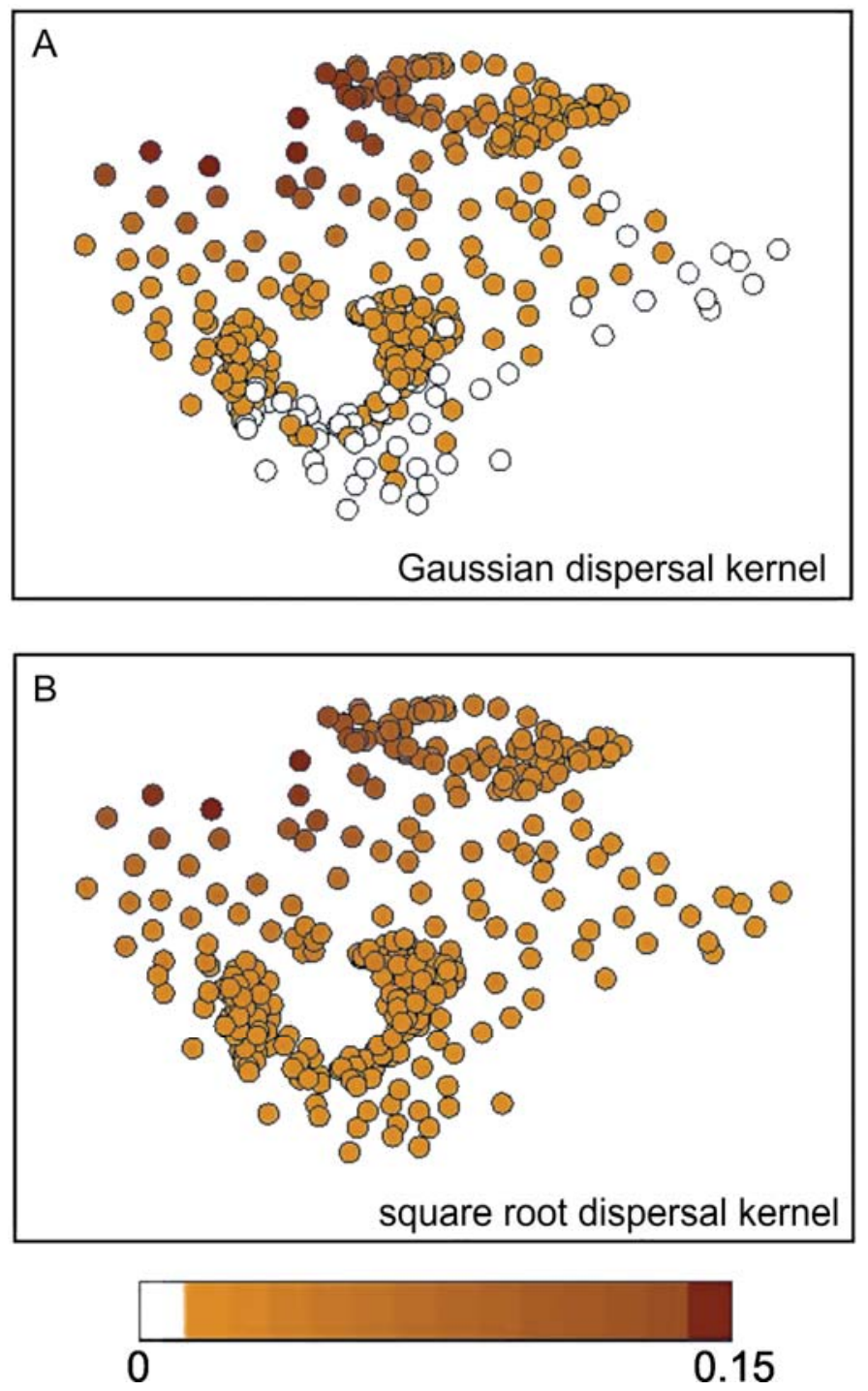

Fig. 3. Maps showing the probability of being infected for each host, across 1,500 epidemic realizations for $\mathbf{A}$, the Gaussian dispersal kernel and $\mathbf{B}$, the square root dispersal kernel. The white dots are hosts that have not become infected. The darkness of the other hosts increase with the probability of being infected. The intensity of the points increases from gray to black with an increase in the probability. The initial number of exposed hosts equals 10 , with epidemiological parameters $\beta=0.0007, \gamma=0.0005$, and $\theta=0.083$. the source of initial inoculum. We use the minimum distance at which host plants are located from the source of initial inoculum as the starting distance for stratification. Furthermore, we consider proportional allocation of the sample scheme within strata. Whereby for sample size of $n$, the contribution of each stratum to the sample scheme equals

$$
\frac{\hat{N}_{i}}{N} \cdot n
$$

where $\hat{N}_{i}$ is the number of plants in the $i$ th stratum and $N$ is the total number of plants, with all sample elements chosen at random.

Effect of the epidemiological parameters and time on the detection probability. We also determine the dependence of the detection probability on the numeric value of the epidemiological parameters, the dispersal kernel, and the time elapsed since first introduction of the disease in the nursery for distance-based sampling. We apply sample schemes consisting of the $n$ hosts closest to the source of infection to sets of realizations of the epidemic developed with different values of the epidemiological parameters. This allows us to establish which epidemiological parameters are essential in determining the efficiency of the sampling method in detecting disease. We also apply the distancebased sampling schemes to realizations for which the disease had been present for either $T=50,100,150$, and 200 days before sampling was applied.

\section{RESULTS}

As default, we applied the sampling methods to realizations of the epidemic at $T=100$ days unless otherwise stated. The Gaussian kernel gives rise to more localized dispersal than the square root kernel (Fig. 3). We find that the optimized sample scheme results in high detection probabilities of disease outside the eradication zone. The detection probabilities obtained with distance-based sampling were usually close to those obtained with optimized sampling. The detection probabilities obtained with sampling based on the probability maps were somewhat lower than those obtained for the above sampling methods (Fig. 4). We only show the results of the detection probability obtained for the epidemic generated with the Gaussian kernel (Fig. 4), but the results are qualitatively similar when the square root dispersal kernel is assumed. The three sampling methods (simulated annealing, probability map sampling, and distance-based sampling) above result in a larger detection probability when compared with random sampling and stratified random sampling (Fig. 4). Stratified sampling gives the lowest detection probability overall compared with the other methods. We find that the locations of sampling sites allocated within the optimum sample scheme overlap with most locations within the sample schemes for distance-based sampling and probability map-based sampling, which explains why the detection probability obtained for these three sampling methods differs so little from each other (Fig. 5). Other sets of parameter values (in the range as used in Fig. 6) result in very similar correspondence between the sampled hosts in the three sampling methods (data not shown). If the nursery/garden center is placed at different locations within the host distribution than shown here, similar results as those described above are obtained (data not shown).

Since our results show that the probability of detection of distance-based sampling is close to the maximum detection probability as resulting from the optimization method, we show here only the effect of the epidemiological parameters on the efficiency obtained with distance-based method. The efficiency of disease detection outside the eradication zone obtained with distance-based sampling decreases considerably as the latent period increases. For example, for a sample scheme of size $n=20$, when 
the latent period is double the default value of 12 days, we find a $16 \%$ decrease in the detection probability. When the latent period is increased four times the effect is greater, as we find a $68 \%$ decrease in the detection probability (Fig. 6A). The detection probability is influenced to a lesser extent by the value of the transmission rate than the latent period, but an increase in the transmission rate also leads to an increase in the detection probability (Fig. 6C). Decreasing the infectious period, $1 / \gamma$, hardly influences the value obtained for the detection probability with distance-based sampling (Fig. 6D), whereas an increase in the time that the disease is present before it is detected and locally eradicated in the nursery greatly influences the obtained detection probability of disease (Fig. 6B).

\section{DISCUSSION}

Some plant pathogens invade new areas by escaping from trade networks. $P$. ramorum, causing sudden oak death, is a recent example. In such cases early detection is essential in reducing their potential damage to the environment as well as the investment required for effective control. We showed how optimized sampling can be used to derive the optimal sampling scheme for early detection of invasive pathogens using a general numerical optimization method. Although simulated annealing is new to plant pathology, it is widely used in geostatistics $(22,28)$. For a more detailed description of its use in that context we refer to Lark (16). Applying the numerical method to the example of $P$. ramorum combined with the specified host distribution, we have shown that (i) optimized sampling can be applied to detect invasive pathogens, and (ii) we can derive optimum sample schemes for disease detection by combining the method with an epidemiological model. The optimum sample scheme derived using simulated annealing guarantees the highest probability of disease detection outside an eradication zone (15). It does however require an epidemic simulation model as well as the optimization

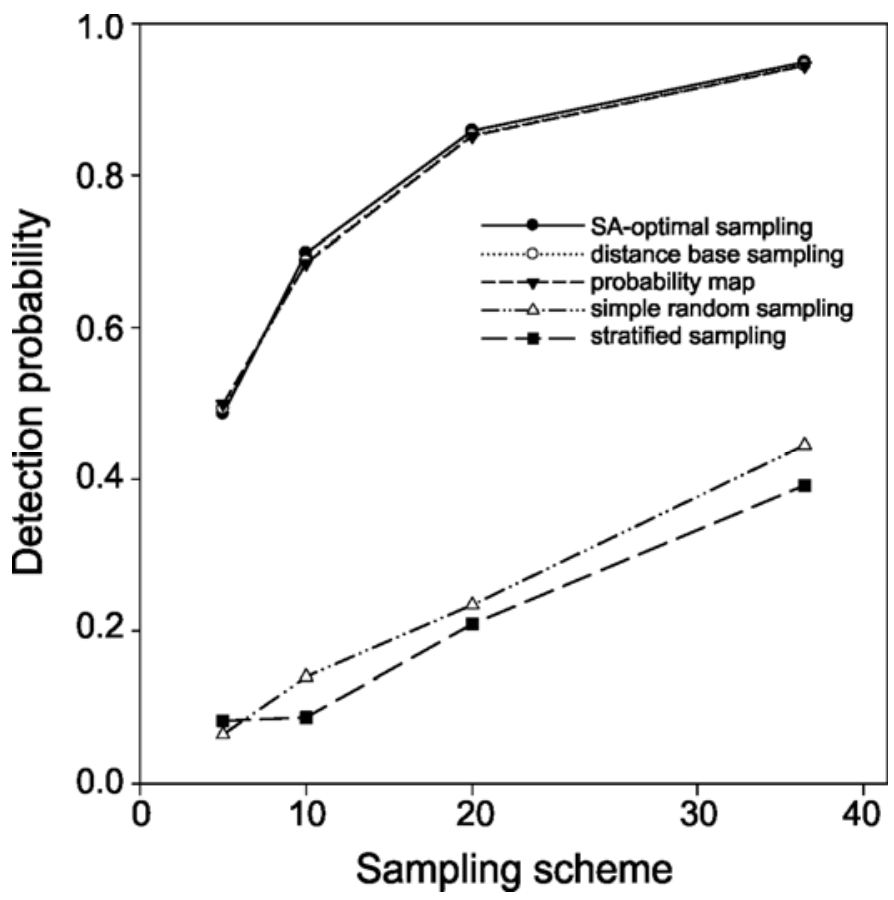

Fig. 4. The probability of detecting the pathogen for the optimized sampling method compared with the sample scheme based on the probability maps (plotted in figure 3), distance-based sampling, simple random sampling, and stratified sampling, applied to realizations of the epidemic at $T=100$ days with the Gaussian dispersal kernel. As the difference in the values of the detection probabilities obtained with the different methods is very small, the graphs for three are almost not distinguishable. Parameters are $\beta=0.0007, \gamma=$ 0.0005 , and $\theta=0.083$. routine to be available to the researcher. Furthermore, maps of the host distribution and the values of the model parameters (transmission rate, latent period, infectious period, and spore dispersal distance) are needed. These are available in some cases and not in
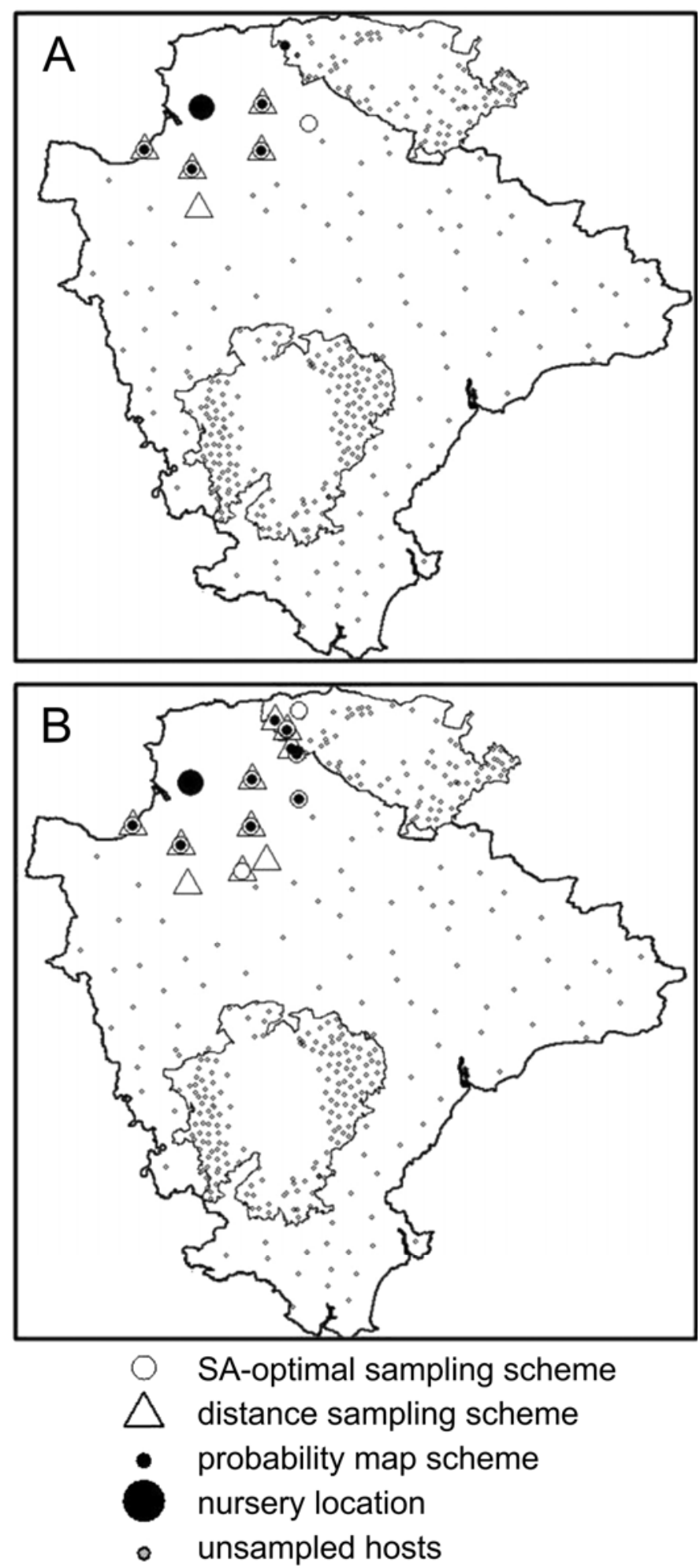

Fig. 5. The sample scheme, i.e., the set of hosts sampled, obtained for a putative epidemic generated with the Gaussian dispersal kernel for A, sample size $n=5$ and $\mathbf{B}$, sample size $n=10$. The large black dot is the location where the epidemic is initiated and the small gray dots are the unsampled hosts. The white dots, white triangles, and small black dots are the elements within the optimum sampling scheme, the distance-based sampling scheme, and the probability map sampling scheme, respectively. The epidemiological parameters are $\beta=0.0007, \gamma=0.0005$, and $\theta=0.083$. 
others. We used the optimal sample plan found by simulated annealing to assess the performance of other, simpler to use, methods to design sampling plans. This allows to derive "rules of thumb" that can be applied in practical field situations. We have shown that in the case investigated in this paper the performance of probability map-based sampling and distance-based sampling is very close to that of the optimal sampling plan. Simple random sampling and stratified sampling give the lowest detection probabilities overall (Fig. 5).

Sampling for detection of invasive plant pathogens has received little attention compared with the extensive work done on sampling to detect rare or invasive animals and plants (7). In an empirical study on detection of invasive plants, Huebner (11) tested four sampling strategies: systematic-plot sampling, stratified-random plot sampling, the modified Whittaker method, and the timed-meander method. Timed-meander sampling outperformed the other strategies considered, with stratified sampling performing second best. The timed-meander method involves thoroughly walking through smaller sites within the larger study area for one hour noting the time every $10 \mathrm{~min}$ as new species were counted. The method sampled $100 \%$ of each site. The timedmeander method shows similarities with the optimization method we applied. Both methods take into consideration the complete sampling area, but optimized sampling allows us to input prior knowledge, i.e., epidemiological information, and gives us a set of optimum locations to sample for maximum detection. In a theoretical study into sampling for detection, Been and Schomaker (3) developed a simulation model to generate population densities for potato cyst nematodes on which they tested several grid-based sampling methods. They found that increasing the grid length and width, consequently increasing the sampled area, led to an increase in the average detection probability. Additionally, they showed that the detection probability obtained with the computer program SAMPLE, which derives sampling recommendations based on characteristics of the infestation foci, e.g., its size and population density, was much higher than that obtained with the statutory soil sampling methods. Clearly, devising sampling strategies based on some knowledge of the dynamics of the species to be sampled increased the detection probability. We reach the same conclusion for detection of invasive disease.
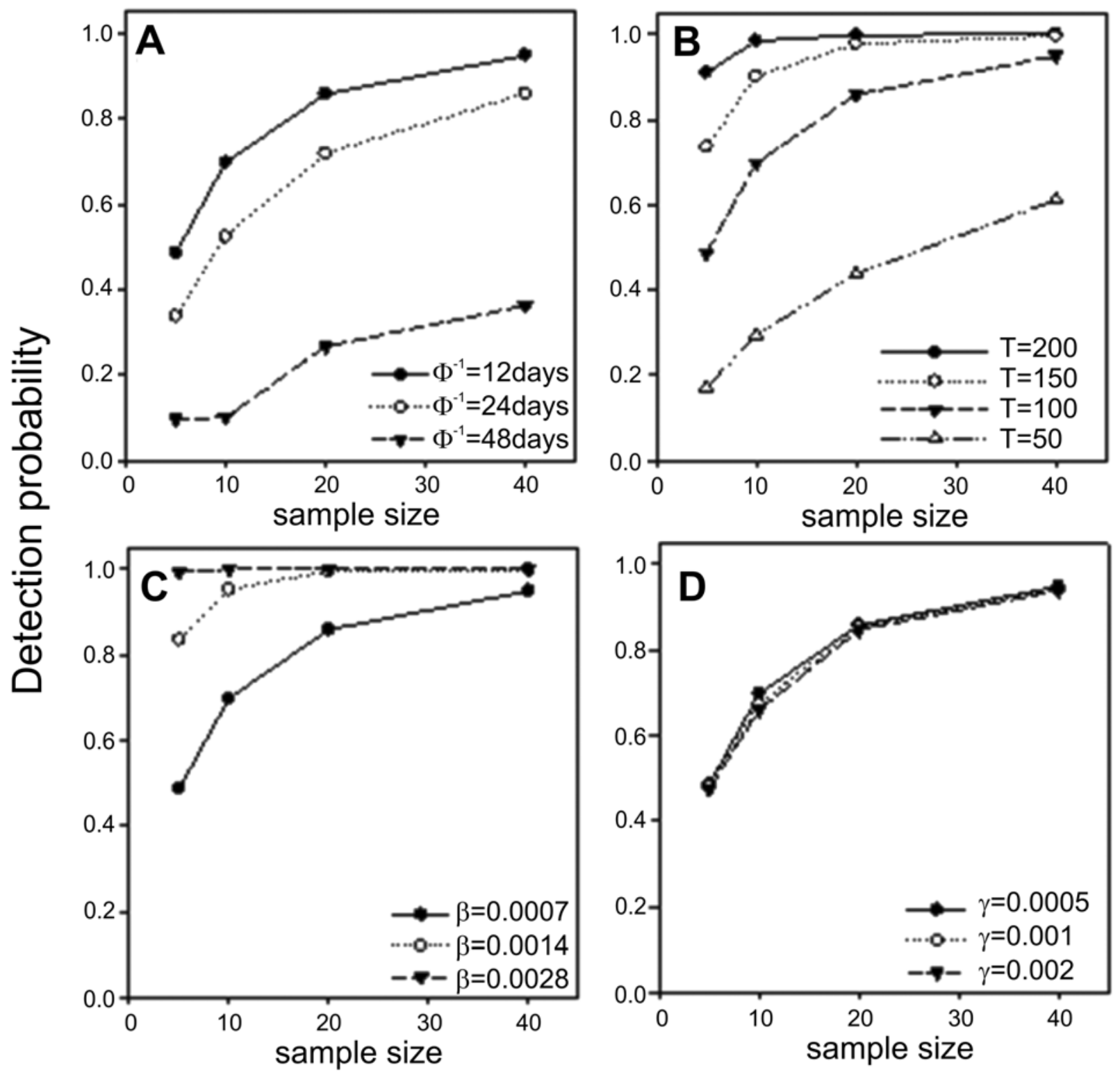

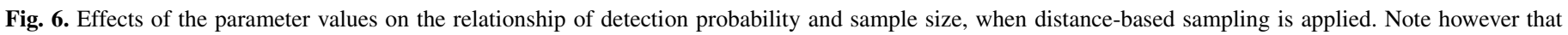
using simulated annealing the resulting sampling plan is marginally better than the distance-based sampling plan. The difference between the two methods is similar as in Figure 4, which is the reason we did not include the results in this figure. A, Latent period, 1/ $\theta$, B, time elapsed since the start of the outbreak T, C, transmission rate, $\beta$, and $\mathbf{D}$, infectious period, $1 / \gamma$. 
Specifically, we have shown that our optimum spatial sampling method can be used to develop sampling schemes that maximize the detection probability. We have also shown the use of these optimized sampling schemes in assessing the effectiveness of other types of sampling methods with the aim of developing ruleof-thumb sampling methods that are easily implemented in practice. In doing this, we have shown that if the location of the source of infection is known, distance-based sampling is a simple and efficient method with a probability to detect disease that is close to the maximum as given by optimized spatial sampling.

\section{ACKNOWLEDGMENTS}

I. Demon is funded by the Department for Environment Food and Rural Affairs (DEFRA), UK. F. van den Bosch receives support from the Biotechnological and Biological Research council (BBSRC) of the United Kingdom. C. A. Gilligan also gratefully acknowledges the support of a BBSRC Professorial Fellowship. We thank M. Lark and S. Parnell for helpful discussion and input in the methods applied in this study.

\section{LITERATURE CITED}

1. Appiah, A. A., Jennings, P., and Turner, J. A. 2004. Phytophthora ramorum: One pathogen and many diseases, an emerging threat to forest ecosystems and ornamental plant life. Mycologist 18:145-150.

2. Barr, K. J., and Asher, M. J. C. 1992. The host range of Polymyxa betae in Britain. Plant Pathol. 41:64-68.

3. Been, T. H., and Schomaker, C. H. 2000. Development and evaluation of sampling methods for fields with infestation foci of potato cyst nematodes (Globodera rostochiensis and G. pallida). Anal. Theor. Plant Pathol. 90:647-656.

4. Brasier, C. M. 2008. The biosecurity threat to the UK and global environment from international trade in plants. Plant Pathol. 57:792-808.

5. Brasier, C. M., and Gibbs, J. N. 1975. Highly fertile form of the aggressive strain of Ceratocystis ulmi. Nature 257:128-131.

6. Brasier, C. M., and Jung, T. 2006. Recent developments in Phytophthora diseases of trees and natural ecosystems in Europe. Pages 5-16 in: Proceedings of the Third International IUFRO Working Party S07.02.09 Meeting. C. M. Brasier, T. Jung, and W. Oßwald, eds. IUFRO, Freising, Germany.

7. Burnham, K. P. 2004. Sampling Rare or Elusive Species: Concepts, Designs and Techniques for Estimating Population Parameters. W. L. Thompson, ed. Island Press, Washington.

8. Commission, Forestry. 2009. Report on the Forestry Commission ReSurvey of Woodlands 2008 to Assess the Level of Incidence of Phytophthora ramorum and Phytophthora kernoviae in Woodlands in England, Wales and Scotland. Forestry Commission, Edinburgh

9. Fry, W. E., Goodwin, S. B., Dyer, A. T., Matuszak, J. M., Drenth, A., Tooley, P. W., Sujkowski, L. S., Koh, Y. J., Cohen, B. A., Spielman, L. J., Deahl, K. L., Inglis, D. A., and Sandlan, K. P. 1993. Historical and recent migrations of Phytophthora infestans: Chronology, pathways, and implications. Plant Dis. 77:653-661.
10. Garbelotto, M. 2004. Sudden oak death: A tale of two continents. Outlooks Pest Manage. 15:85-89.

11. Huebner, C. D. 2007. Detection and monitoring of invasive exotic plants: A comparison of four sampling methods. Northeastern Nat. 14:183-206.

12. Hughes, G., Madden, L. V., and Munkvold, G. P. 1996. Cluster sampling for disease incidence data. Phytopathology 86:132-137.

13. Jenkins, P. T. 1996. Free trade and exotic species introduction. Conserv. Biol. 10:300-302.

14. Jones, D. R., and Baker, R. H. 2007. Introductions of non-native plant pathogens into Great Britain, 1970-2004. Plant Pathol. 56:891-910.

15. Kirkpatrick, S., Gelatt, C. D., and Vecchi, M. P. 1983. Optimization by simulated annealing. Science 220(4598):671-680.

16. Lark, R. M. 2002. Optimized spatial sampling of soil for estimation of the variogram by maximum likelihood. Geoderma 105(1-2):49-80.

17. Madden, L. V., and Hughes, G. 1999. An effective sample size for predicting plant disease incidence in a spatial hierarchy. Phytopathology 89:770-781.

18. Madden, L. V., and Hughes, G. 1999. Sampling for plant disease incidence. Phytopathology 89:1088-1103.

19. Madden, L. V., Hughes, G., and Munkvold, G. P. 1996. Plant disease incidence: Inverse sampling, sequential sampling, and confidence intervals when observed mean incidence is zero. Crop Prot. 15:621-632.

20. Madden, L. V., Hughes, G., and van den Bosch, F. 2007. Spatial aspects of epidemics-II: A theory of spatio-temporal disease dynamics. Pages 211233 in: The Study of Plant Disease Epidemics. The American Phytopathological Society, St. Paul, MN.

21. Madden, L. V., Hughes, G., and van den Bosch, F. 2007. Measuring plant diseases. Pages 11-31 in: The Study of Plant Disease Epidemics. The American Phytopathological Society, St. Paul, MN.

22. Marchant, B. P., and Lark, R. M. 2007. Optimized sample schemes for geostatistical surveys. Math. Geol. 39:113-134.

23. Mehta, S. V., Haight, R. G., Homans, F. R., Polasky, S., and Venette, R. C. 2007. Optimal detection and control strategies for invasive species management. Ecol. Econ. 61:237-245.

24. Peterson, J. T., and Dunham, J. 2003. Combining inferences from models of capture efficiency, detectability, and suitable habitat to classify landscapes for conservation of threatened bull trout. Conserv. Biol. 17:1070-1077.

25. Pimentel, D., Lach, L., Zuniga, R., and Morrison, D. 2000. Environmental and economic costs of nonindigenous species in the United States. Bioscience 50:53-64.

26. Rice, J. A. 1994. The exponential density. Pages 23-31 in: Mathematical Statistics and Data Analysis. J. Burger, ed. Wadsworth Publishing Co., Belmont, CA.

27. Slawson, D., Bennett, L., Parry, N., and Lane, C. 2006. The current situation with Phytophthora ramorum in England and Wales. Pages 455457 in: Sudden Oak Death Second Science Symposium: The State of Our Knowledge. S. J. Frankel, P. J. Shea, and M. I. Haverty, eds. Gen. Tech. Rep. PSW-GTR-196, U.S. Dep. Agric., Forest Service, CA

28. van Groenigen, J. W. 1999. Constrained Optimisation of Spatial Sampling: A Geostatistical Approach. Soil Sciences, Wageningen Agricultural University, Wageningen.

29. Venette, R. C., Moon, R. D., and Hutchison, W. D. 2002. Strategies and statistics of sampling for rare individuals. Annu. Rev. Entomol. 47:143174. 